Usages du français et pratiques d'enseignement en Europe balkanique, centrale et orientale - Grèce,

Serbie, Bulgarie, Moldavie, Hongrie, Allemagne, Russie - XVIIIe - XXe siècles

\title{
Les écoles françaises en Bulgarie (1864-1948)
}

\author{
Julieta Velichkova-Borin
}

\section{(2) OpenEdition Journals}

\section{Édition électronique}

URL : https://journals.openedition.org/dhfles/4239

DOI : $10.4000 /$ dhfles.4239

ISSN : 2221-4038

\section{Éditeur}

Société Internationale pour l'Histoire du Français Langue Étrangère ou Seconde

\section{Édition imprimée}

Date de publication : 1 juin 2015

Pagination : 171-191

ISSN : 0992-7654

Référence électronique

Julieta Velichkova-Borin, «Les écoles françaises en Bulgarie (1864-1948)», Documents pour l'histoire du français langue étrangère ou seconde [En ligne], 54 | 2015, mis en ligne le 01 janvier 2018, consulté le 30 mars 2023. URL : http://journals.openedition.org/dhfles/4239 ; DOl : https://doi.org/10.4000/ dhfles.4239

Ce document a été généré automatiquement le 30 mars 2023 


\title{
Les écoles françaises en Bulgarie (1864-1948)
}

\author{
Julieta Velichkova-Borin
}

1 Les écoles françaises en Bulgarie (1864-1948) sont une œuvre de la France catholique qui se maintient dans le cadre d'une France devenue en 1905 une république laïque. Or, pour des raisons d'ordre politique, l'activité des catholiques en Bulgarie pour la période qui va de la libération de la Bulgarie du joug ottoman (1878) jusqu’à la fin de la Seconde Guerre mondiale a longtemps été un sujet tabou dans l'historiographie bulgare depuis 1944 (Eldarov 2002 : 3). Ainsi, entre 1944 et 1989, une communication du professeur Nikolay Kochev, semble être la seule publication qui parle de l'Église catholique (Kotchev 1988 : 23-31). Les années 1990 semblent plus riches : une quinzaine de titres monographies et articles - doivent combler les lacunes. Malgré les efforts d'auteurs comme S. Eldarov, V. Tapkova-Zaïmova, L. Guenova, R. Zaïmova, la recherche historique en Bulgarie est toujours redevable aux missions catholiques, à leurs activités parmi les Bulgares et surtout à ce "phénomène de grande ampleur» (Thobie 2010 : 140) que sont les collèges français, créés par différentes congrégations catholiques et soutenus par le service spécial des Affaires étrangères de France, appelé Les Euvres, malgré la séparation de l'Église et de l'État, en 1905.

2 Il faut noter que la Bulgarie fait partie des pays dans lesquels la majorité de la population est rattachée à l'orthodoxie. Lors du recensement de 2011, alors que la déclaration d'appartenance religieuse par les citoyens bulgares n'était pas obligatoire, $59,4 \%$ se sont identifiés comme chrétien orthodoxe et $7 \%$ comme musulman. Plusieurs autres groupes religieux sont présents depuis plusieurs siècles sur les terres bulgares : juifs, catholiques, protestants et arméniens; chacun de ces groupes constitue néanmoins moins de $1 \%$ de la population (The National Statistical Institute of Bulgaria 2011).

3 Même si les Bulgares sont majoritairement orthodoxes, leur histoire religieuse, politique et culturelle est dès le XVII ${ }^{e}$ siècle marquée par la présence des diocèses catholiques de Sofia, la capitale bulgare et Plovdiv, la plus importante ville dans le sud de Bulgarie, fondé en 1601 et de Nikopol, ville bulgare au bord du Danube, fondé en 
1648 par les franciscains. C'est de cette époque que date la première école catholique ${ }^{1}$, créée à Chiprovtsi en 1624, une ville dont on retiendra l'échec du soulèvement contre la domination ottomane en 1688.

4 Les Frères franciscains observants sont en effet les premiers missionnaires catholiques à s'installer dans les terres bulgares (partie intégrante de l'Empire ottoman jusqu'à 1878). Les premiers capucins arrivent deux siècles plus tard, le 21 mars 1841, à Plovdiv et les débuts de leur activité parmi les Bulgares coïncident avec les réformes timides, entamées dans l'Empire ottoman en 1839 avec le Hatt-i Sharif de Gülhane. Ils sont suivis par les Augustins de l'Assomption et les Oblates de l'Assomption, dans les années 1860. Les Sœurs de Saint Joseph de l'Apparition s'installent à Plovdiv dès 1866. Les Sœurs de Notre-Dame de Sion remplacent les Oblates de l'Assomption à Roussé, ville bulgare au bord du Danube, après leur départ en 1896.

5 Les congrégations catholiques présentes en Bulgarie affichent des objectifs différents. Par exemple, pour les Augustins de l'Assomption du Père d'Alzon c'est la conversion de la Russie par la Bulgarie qui importe (Brombart 2010 : 223). En revanche les Sœurs Oblates de l'Assomption, fondées pour seconder les Assomptionnistes en Bulgarie, se donnent une tâche missionnaire, œcuménique, caritative, éducative et sociale. L'éducation des jeunes filles par les religieuses de la congrégation Notre-Dame de Sion et auprès des Sœurs de Saint Joseph de l'Apparition est fort renommée et donc recherchée. Il faut souligner à ce sujet l'importance politique que représente la protection française des catholiques et des établissements religieux en Orient $^{2}$.

6 C'est dans le domaine de l'éducation et de l'enseignement que les congrégations catholiques en Bulgarie atteignent leurs réussites les plus impressionnantes. « Défendre la vérité, l'enseigner à tout âge, la communiquer au plus grand nombre ", telle était, selon le P. d'Alzon, la cause de l'enseignement catholique. Les congrégations catholiques jouent un rôle primordial dans la propagation de la langue et de la culture françaises à l'étranger. Leurs actions hors de France sont toujours placées sous l'égide du ministère des Affaires étrangères (Zaïmova 2010 : 286). L'enseignement du français et l'éducation "à la vérité » dont les congrégations font la promotion dans leurs écoles correspondent à l'idéologie et aux valeurs de l'enseignement républicain chargé de contribuer à l'unification et au progrès de la nation française.

\section{Les écoles françaises}

7 En 1862, le P. d'Alzon envoie Victorin Galabert ${ }^{3}$ à Constantinople jeter les bases d'une fondation de l'Assomption pour préparer les voies d'une union des Bulgares à l'Église catholique. En arrivant en Bulgarie, son premier acte est de créer en janvier 1864 une petite école primaire Saint André à Philippopoli (Plovdiv), qui, en 1884, donnera naissance au collège Saint Augustin (Périer-Muzet 2000 : 1189-1190; Galabert 2000). C'est sur les instances du P. Galabert, désireux d'être épaulé pour la mission par des religieuses, qu'en mai 1865, le P. d'Alzon fonde la Congrégation des Oblates.

8 En lisant aujourd'hui les recommandations du P. Galabert, on se rend compte, que rien n'est acquis d'avance :

Avant tout donc les PP. Augustins de l'Assomption agiront avec un grand esprit de désintéressement et sauront éviter tout ce qui pourrait faire soupçonner en eux l'idée de vouloir s'imposer, dominer le clergé oriental 
catholique et s'attribuer la direction des affaires. Pour gagner les populations et faire tomber peu à peu des préventions absurdes mais profondément enracinées, ils s'adonneront aux œuvres de charité, se montreront très prévenants envers tout le monde, supporteront patiemment les grossièretés, seront indifférents aux injures et ne se laisseront décourager ni par l'ingratitude ni par l'insuccès. Ils sauront édifier par de bons exemples, seront toujours prêts à rendre service, à donner des conseils demandés et ne commanderont jamais. En toutes circonstances ils s'effaceront pour laisser aux autres l'honneur de résultats obtenus souvent malgré leur opposition [...] Les Augustins de l'Assomption doivent s'attendre à de nombreux insuccès. (Brombart $2010: 223$ )

Les réflexions du ministre de France à Sofia, M. Allizé, formulées à peu près une cinquantaine d'années plus tard, vont dans le même sens :

\begin{abstract}
Notre intervention en ce qui concerne nos établissements scolaires religieux est particulièrement délicate en Bulgarie parce que nous nous trouvons dans un pays de chrétienté, où le sentiment religieux est étroitement connexe au sentiment national et où les établissements scolaires de l'État sont déjà très développés ${ }^{4}$.
\end{abstract}

Outre les différences religieuses et culturelles, les conditions politiques sont très peu favorables à l'implantation durable des écoles françaises dans la vie sociale et culturelle des Bulgares. Cette implantation semble pourtant plus ou moins réalisée à la veille de la Première Guerre mondiale. Les enseignants du collège Saint Augustin à Philippopoli (Plovdiv) tirent orgueil de la plupart de leurs anciens élèves devenus sous-officiers, médecins, ingénieurs, professeurs, commerçants etc. (Fleury 2003 : 79-80). Malgré ces bons résultats et la mobilité sociale garantie des élèves des collèges français, une première expulsion des enseignants français s'est faite «dans la douleur et dans la dureté », en 1915 car la Bulgarie faisait partie de la Triplice ${ }^{5}$ pendant la Grande Guerre. Et dans le rapport adressé au ministre français à Sofia par les expatriés français, on remarque l'opposition entre la bonté des prêtres Assomptionnistes et la dureté des gouvernements envers eux, et cela malgré une attitude favorable des religieux envers le peuple bulgare durant les deux guerres Balkaniques (1912-1913), lorsqu'ils se sont "dévoués corps et âme pour les Bulgares ", " en mettant leurs établissements à la disposition de la Reine et en aidant la Croix-Rouge française à y installer une ambulance ». Même le collège Saint Augustin à Plovdiv est alors transformé en hôpital. Pourtant les sœurs de Varna seront internées dans des conditions précaires; les religieux et religieuses français expulsés et réfugiés en Roumanie seront maltraités en attendant leur rapatriement par la Russie, la Norvège et l'Angleterre; leurs écoles seront fermées. Tout cela parce que les pères soupçonnés d'espionnage étaient accusés d'avoir aidé la flotte russe pendant la Première Guerre mondiale'

11 Malgré des considérations et des actes d'ordre politique, moral et religieux très souvent défavorables à leur égard, pendant la Première et la Seconde Guerre mondiale, les écoles françaises continuaient à servir pour les Bulgares de «modèle de conduite qui comprenait aussi la tolérance dans les relations avec les 'différents' ». Ainsi, pendant la Seconde Guerre mondiale, alors que les juifs n'ont pas le droit de fréquenter d'autres écoles que des écoles juives, ceux-ci sont accueillis au pensionnat français de Roussé pour y faire leurs études une fois que les autres élèves ont terminé leurs leçons (Rochkéva, Tzvetkova 2008 : 17). 
12 L'école est un lieu de travail, de continuité, de transfert et d'interaction. Elle peut être observée et évaluée sous différents aspects. Pour les 17 écoles $^{7}$ catholiques françaises (dix écoles primaires, un séminaire et six collèges) c'est d'abord la qualité de l'enseignement qui est remarquable, aussi bien par les pratiques scolaires que dans les manuels utilisés. Chaque école française développe des activités culturelles et en particulier musicales. Si chaque école a son piano, à l'école Notre-Dame de Sion il y en avait même deux, pianos à queue. Chaque collège avait sa fanfare, sa chorale et ses uniformes (un pour les sorties et un pour tous les jours, un pour l'été et un pour l'hiver). Chaque collège a son musée, ses salles d'études et ses laboratoires, sa bibliothèque, son pensionnat (sauf le collège de garçons SS. Cyrille et Méthode à Sofia qui n'avait que des externes). Outre les laboratoires et les bibliothèques, les écoles disposaient de terrains de sport, louaient des villas pour les vacances et assuraient à leurs élèves et professeurs, sans doute, les meilleures conditions de travail que l'on pouvait désirer à l'époque. L'objectif essentiel des religieux/religieuses était de stimuler l'esprit créateur et l'imagination des élèves, en proposant des journaux rédigés par les élèves, des concerts et des compétitions sportives organisés pour servir des causes nobles de bienfaisance. La Société de Saint Vincent de Paul (élèves du collège français de Plovdiv) existe depuis l'ouverture du collège en 1884; elle distribuait chaque semaine du pain et autres secours aux pauvres de la ville pour lesquels elle donnait son concert annuel. Le quotidien laborieux est, entre autres, rythmé par les concerts et les loteries des pauvres, les matinées littéraires, les séances musicales et récréatives, les fêtes sportives, le travail sur les magazines illustrés manuscrits.

Les activités para scolaires ne doivent pas cacher que l'accent est mis sur les études, l'instruction et l'éducation. L'attrait de ces écoles françaises est d'autant plus fort que les diplômes délivrés par les collèges (certificats de maturité) étaient reconnus également par le gouvernement bulgare et par la France; ils permettaient donc de continuer les études dans les universités des deux pays et dans certaines universités européennes. À la valeur pédagogique de ces écoles s'ajoute une valeur sociale essentielle, car elles sont un facteur de promotion au sein de la société bulgare. Mais le succès de ces écoles françaises est aussi dû surtout aux enseignants, à leur qualification et qualités personnelles, à leur dévouement.

\section{Les enseignants}

Bien que religieux ou religieuses, les enseignants dans les écoles françaises sont des professionnels, formés à leur tâche précise. Leurs diplômes de professeurs sont délivrés par un Institut d'études supérieures, établi spécialement à Constantinople en 1895 pour les jeunes missionnaires français. Pour être admis dans cet Institut, les élèves doivent avoir achevé leurs études secondaires conformes aux programmes français (latin, grec, sciences, littérature).

L'Institut d'études supérieures de Constantinople leur donne une formation pédagogique plus spéciale aux écoles d'Orient. Leurs études, secondaires en France, supérieures en Orient, les rendent plus que compétents comme professeurs de nos écoles en général et en particulier de notre École de Varna, où l'enseignement s'est borné jusqu'ici aux éléments de la langue française, bien que nous désirions développer cet enseignement, dès que nous aurons construit le pensionnat projeté depuis plusieurs années ${ }^{8}$. 
l'article 9 stipule que :

Les nominations de professeurs et directeurs étrangers seront soumises, par l'intermédiaire de la légation de France, à l'approbation du Ministère de l'Instruction publique, sur le vu d'un certificat de moralité délivré par le gouvernement de la République. Les directeurs et professeurs devront présenter, en outre, un certificat d'aptitude à l'enseignement, délivré par le Gouvernement français 9 .

Cette Convention marque un changement important pour le recrutement du personnel qui coïncide avec le transfert de l'Institut de Constantinople à Bucarest sous le nom d'Institut français d'études byzantines. Le 13 novembre 1936, le ministère des Affaires étrangères (Service des œuvres françaises à l'étranger) entreprend un recensement du personnel enseignant français (laïque et religieux) à l'étranger, en envoyant une lettre aux ambassades. Le 2 septembre 1937, le ministre plénipotentiaire de la République française en Bulgarie retransmet le questionnaire aux écoles françaises en Bulgarie. Les informations recensées sont l'âge, le domicile, les diplômes, la durée de l'enseignement donné dans le pays, la nature de l'enseignement, l'établissement où cet enseignement est donné. Les enseignants religieux dans les neuf écoles françaises en 1937 sont au nombre de 85. La majorité des enseignants sont voués au célibat. Étant des missionnaires catholiques, ils accomplissent leur tâche avec compétence et dévouement. Depuis leur installation en Bulgarie ils sont sensibles à la réceptivité du milieu d'accueil, sans négliger non plus les changements politiques en France et la promulgation de nouvelles lois scolaires. Dans une déclaration, signée le 18 décembre 1882 à Varna, le sous-préfet de la mission latine dans cette ville au bord de la mer Noire écrit : «À cause de la différence des croyances des élèves [...] toute idée religieuse sera éloignée de l'établissement $»^{10}$.

\section{Les élèves}

En ce qui concerne les élèves, il s'agit du public le plus disparate possible autant sur le plan religieux que sur le plan ethnique.Le recrutement se fait comme à l'époque de l'Empire ottoman : chaque enfant, nonobstant sa religion, a le droit de s'y inscrire. Le catholicisme n'est pas imposé, et toujours comme à l'époque ottomane, les élèves travaillent dans une ambiance de tolérance religieuse. On accueille des Bulgares orthodoxes et catholiques, des juifs, des Arméniens, à côté des enfants du personnel des missions diplomatiques et commerciales dans le pays, donc étrangers. Par exemple, en 1917, le nombre des élèves orthodoxes de Saint Joseph de l'Apparition à Sofia est de 280, les catholiques étrangères sont 128 , et 192 sont juives (Zaïmova $2010: 4$ ).

Les effectifs de ce même collège de jeunes filles augmentent constamment et en 1941/1942, sept ans avant sa fermeture, la répartition des 791 élèves par nationalité est la suivante : 384 Bulgares, 289 Israélites, 30 Arméniennes, 88 diverses. La même année scolaire, la proportion des nationalités parmi les 652 élèves du collège français de garçons SS. Cyrille et Méthode à Sofia (Lozénetz) est la suivante : 370 Bulgares, 219 Israélites, 30 Arméniens, 33 divers. Même si des familles protestantes et musulmanes, moins nombreuses toutefois, ont également des raisons d'envoyer leurs enfants dans les collèges catholiques français. Les effectifs multiethniques de ces collèges se 
multiplient attirés soit par l'esprit universel et la souplesse administrative des œuvres missionnaires catholiques soit par le prestige de la langue et de la culture françaises dans le royaume de Bulgarie dont la constitution garantit la liberté totale des établissements religieux et d'enseignement et les collèges catholiques français jouissent $\mathrm{du}$ soutien du prince régnant, Ferdinand de Saxe-Cobourg-Gotha et de son épouse, Marie-Louise de Bourbon-Parme.

\section{Les programmes}

Dans les écoles primaires « le programme suivi est celui des écoles primaires en France, la langue bulgare y est obligatoire $»^{11}$. Le programme laïque scolaire respecte les exigences du ministère de l'Instruction publique. L'enseignement est marqué par le respect de la religion. Le terme qui s'impose est celui de "tolérance». On insiste surtout sur la moralité de l'individu, en essayant de lui inculquer l'idée qu'il ne peut pas vivre tout seul, qu'il a besoin des autres. Mais vivre avec les autres signifie avant tout être tolérant. C'est cette approche qui permet la coexistence par excellence dans ces écoles des différentes nationalités et religions.

20 Le but du collège français Saint Augustin à Plovdiv (1884-1948) est de procurer aux familles aisées le moyen de faire donner à leurs enfants l'instruction et l'éducation telles qu'on les donne dans les collèges de France. Il y a dans l'établissement deux divisions : le grand et le petit collège. Une classe « spéciale » est destinée aux élèves qui ont déjà terminé leurs études primaires, mais qui ne connaissent pas suffisamment la langue française pour suivre les cours réguliers. Selon les résultats obtenus aux examens de fin d'année les élèves passent dans la classe de septième ou en sixième. Tous les cours de cette classe spéciale, expliqués en bulgare par le professeur, ont pour but de donner à l'enfant une connaissance du français suffisante pour entrer dans les autres classes. Le grand collège comprend sept classes. Il est divisé en deux cycles. Le premier cycle est composé des quatre premières classes et se termine par un examen qui permet d'obtenir le Diplôme élémentaire de l'enseignement secondaire qui ne donne pas droit à l'entrée dans les universités, mais témoigne que les élèves sont préparés aux carrières industrielles, commerciales et agricoles. Ils peuvent soit continuer leurs études pour l'obtention du diplôme de fin d'études soit entrer dans la section commerciale. Le deuxième cycle est composé de trois classes: seconde, première et classe de philosophie. À l'issue de cette dernière, il est délivré aux élèves qui subissent avec succès les examens, un diplôme de fin d'études reconnu par le gouvernement français et par le gouvernement bulgare ; avec ce diplôme, les lauréats peuvent entrer dans les diverses universités de France et de Bulgarie. Créée au collège, l'École supérieure de commerce a comme but de former pour le commerce, la banque, l'industrie et les diverses administrations soit des employés supérieurs, soit des directeurs de services, soit des chefs de maison ${ }^{12}$. L'enseignement religieux est réduit à l'instruction religieuse au petit collège et à l'apologétique (en trois parties) au grand collège $^{13}$. En ce qui concerne la langue bulgare, l'enseignement est parallèle à celui de la langue française et conforme aux programmes officiels des écoles primaires et des gymnases en Bulgarie. Notons que cette pratique scolaire représente un intérêt particulier car elle nous invite à revoir certains aspects de l'enseignement de la langue maternelle dans le cadre d'une formation en français destinée à des élèves de langue maternelle bulgare, dans leur propre pays. Aujourd'hui, l'enseignement dans la langue 
maternelle de l'élève est considéré comme essentiel pour améliorer les résultats scolaires. L'utilité de la langue maternelle dans le développement mental, cognitif et émotionnel de l'enfant est indubitable. C'est un moyen important d'améliorer les compétences linguistiques de l'élève. Les compétences solides dans la langue maternelle facilitent l'apprentissage d'une langue seconde. Elles contribuent à l'obtention de meilleurs résultats en lecture et en écriture et permettent de promouvoir la diversité linguistique. Notons aussi que la liberté d'échanger des informations et des connaissances dans de nombreuses langues est susceptible d'enrichir les idées et d'améliorer la manière de penser. Sur le plan linguistique ces collèges bilingues voire multilingues étaient en avance sur leur temps.

Le collège français de garçons SS. Cyrille et Méthode à Sofia (Lozénetz) (1885-1948), dirigé par les Frères des Écoles chrétiennes, tout comme le collège à Plovdiv, donne un enseignement primaire et secondaire (certificat de maturité) et commercial ${ }^{14}$. La même année scolaire, 1941/1942, l'organisation du temps scolaire au collège français de jeunes filles Saint Joseph à Sofia (1872-1948), dirigé par les Sœurs de Saint Joseph de l'Apparition, suit à peu près la même proportionnalité ${ }^{15}$. Cependant, les jeunes filles semblent un peu moins chargées que les garçons si notre observation ne tient pas compte des matières comme les travaux à l'aiguille. Ces cours se faisaient d'ailleurs aussi en français.

\section{L'organisation et les pratiques d'enseignement}

Chaque année scolaire se termine dans les écoles françaises par un examen écrit et oral en toutes les matières devant un représentant de l'Ambassade de France en Bulgarie. Cela permet au ministère des Affaires étrangères français qui assure une subvention annuelle aux écoles françaises d'effectuer un contrôle direct sur les résultats de leur fonctionnement. La fin de l'année scolaire, d'habitude marquée par «une séance récréative ", offerte par les élèves de l'établissement, a lieu vers la fin du mois de juin.

23 Le plan d'études de l'école de Varna prévoit une classe spéciale pour les enfants qui ignorent le français ou le bulgare mais qui ont déjà étudié dans une autre école les autres matières d'enseignement. L'accent est mis sur les exercices de traduction et la conversation française. On applique la méthode d'ollendorff ${ }^{16}$.

À l'École Notre-Dame de Sion à Roussé, les enseignantes écrivent un plan détaillé de la nouvelle leçon au tableau. On interroge chaque élève tous les jours. Dans les années 1920 les élèves sont notées sur 7. Dans les années 1930 et 1940 on introduit une tablette pour chaque classe qui circule pendant les récréations parmi les élèves qui parlent bulgare entre elles, même s'il ne s'agit que d'un mot bulgare. Le soir on punit l'élève qui se retrouve avec la tablette. Il y a une caisse où les élèves qui ont eu la tablette pendant la journée mettent de petites monnaies. L'argent ramassé est utilisé pour aider les élèves pauvres. Cette pratique est adoptée par les deux écoles à Roussé - le pensionnat et l'externat (Rochkéva \& Tzvetkova 2008: 10), mais aussi par le collège de garçons Saint Augustin de Plovdiv, où circule une planchette. Dans ce collège la langue bulgare est autorisée seulement pendant le loisir des élèves - le jeudi et le dimanche de $18 \mathrm{~h} 30$ à $20 \mathrm{~h} 30$ (Fleury 2003 : 151). Rappelons que les religieux/religieuses enseignant dans les écoles catholiques françaises en Bulgarie, expulsés de France après 1905, étaient porteurs de l'idéologie de l'éducation républicaine: la liberté des cultes devait être respectée et depuis l'enquête de l'abbé Grégoire et la nécessité d'universaliser l'usage 
de la langue française il fallait bannir la langue maternelle - qui peut être une langue régionale de France - et ne parler que le français à l'école - une pratique de plus reliant les milieux scolaires en Bulgarie et en France.

\section{La liquidation des écoles françaises en 1948}

Après la Seconde Guerre mondiale la Bulgarie entre dans la sphère d'influence de l'URSS et devient elle-même une "démocratie populaire ", en 1946. Les changements politiques entraînent la fermeture de toutes les écoles étrangères en Bulgarie.

Dans une lettre du 21 mai 1948, Jacques Émile Paris, ministre de France en Bulgarie, écrit à Georges Bidault, ministre des Affaires étrangères :

Au cours d'un dîner qu'il a donné le 18 mai en l'honneur de M. Justin Godart, le Président du Conseil, M. Georges Dimitrov m'a entretenu de la situation des écoles françaises en Bulgarie. C'est là, m'a-t-il dit, le seul point épineux dans nos relations avec la France. Dans aucun autre domaine il n'existe de sujet de litige ou de contestation entre les deux pays. Reprenant l'argument déjà utilisé par M. Kolarov (ma lettre N 27 RC du 7 janvier) il a marqué son étonnement de voir que le Gouvernement français soutenait en Bulgarie des écoles religieuses, alors qu'en France l'enseignement donné par l'État était uniquement laïque. Il faut se rendre compte, a poursuivi M. Dimitrov, que l'existence d'écoles catholiques chez nous consiste une anomalie. L'Église orthodoxe bulgare ne possède pas d'écoles et n'est pas autorisée à en avoir puisque notre constitution dispose que l'enseignement est donné par l'État. [...] En ce qui concerne les immeubles appartenant aux communautés enseignantes ( $c f$. ma lettre N $380 \mathrm{RC}$ du 28 avril) ils ne paraissent pas immédiatement menacés. La seule mesure capable d'en assurer efficacement la protection serait leur cession - réelle ou fictive - à l'État français qui en deviendrait propriétaire aux yeux du Gouvernement bulgare. [...] Je serais très obligé au Département, s'il approuve les suggestions qui précèdent, de bien vouloir en informer les Supérieurs des communautés religieuses enseignantes en Bulgarie (Pères Assomptionnistes, Frères des Écoles chrétiennes, Sœurs de Saint Joseph, Sœurs de Notre-Dame de Sion) et de me donner ses instructions en vue des conversations qui pourraient s'engager dans un avenir prochain avec le Gouvernement bulgare ${ }^{17}$.

Dans sa note verbale du 3 août 1948, le ministère des Affaires étrangères porte à la connaissance de la légation de la République française à Sofia que le Présidium de la Grande assemblée nationale a dénoncé par décret $\mathrm{N}^{\circ} 1144 \mathrm{du} 2$ août 1948, publié au Journal officiel $\mathrm{N}^{\circ} 180$ du 3 août 1948 , la convention scolaire entre la Bulgarie et la France. Cette convention scolaire cessera donc d'être en vigueur neuf mois après la réception de cette communication et notamment au 3 mai 1949. Dans le même numéro du Journal officiel est publié aussi un Ukase sur les écoles étrangères dont l'article premier dispose : 
Les écoles étrangères de toutes catégories et espèces - écoles maternelles, primaires et secondaires, collèges, cours et autres, ouvertes ou subventionnées par des gouvernements de pays étrangers, par des missions et congrégations religieuses ou par des personnes privées, physiques ou juridiques, sont fermées à partir du 1er septembre 1948 ", [exception faite des écoles françaises qui, selon la remarque], peuvent continuer à exister jusqu'à l'expiration du délai de 9 moi à partir de la date de notification de la dénonciation de ladite convention scolaire.

\section{Conclusion}

Comment l'enseignement d'une langue étrangère et l'enseignement donné en une langue étrangère dans des conditions sociopolitiques peu favorables a-t-il été susceptible d'inculquer durablement de valeurs nouvelles? Quelles étaient ces valeurs? Quel était le rôle de la religion dans cette affaire?

Compte tenu des faits, le nombre des élèves bulgares des écoles françaises en Bulgarie s'étant convertis au catholicisme au cours de leur scolarisation sous l'influence de l'école est une quantité négligeable. C'était pourtant un des objectifs essentiels de ces écoles...

Cependant, entre 1864 et 1948, la présence des écoles catholiques françaises en Bulgarie contribue à l'organisation d'un espace social protégé de tolérance, à la fois multiethnique, multiculturel et francophone qui était susceptible de mobiliser les jeunes esprits à apprendre sans endoctriner, sans dogmatiser, tout en stimulant leur bienséance, bienfaisance, bienveillance, leur bonne volonté, le respect d'autrui et l'esprit de corps. On y cultivait la morale individuelle au sens durkheimien. Ces écoles "catholiques " mais aussi «françaises " par la langue d'enseignement et parce que placées sous le protectorat français et subventionnées par la France (Secours aux établissements scolaires religieux et hospitaliers d'Orient, Département des affaires politiques - MAE, Service des œuvres françaises à l'étranger, l'Alliance française, l'Alliance israélite universelle), semblent être à l'origine de la «francophonie d'appel $\aleph^{18}$ de la Bulgarie, aujourd'hui membre de l'OIF et dotée d'un réseau d'écoles secondaires bilingues (bulgare-français) - pépinières d'élites de sensibilité sociale et linguistique spécifiques. Le français enseigné dans les écoles catholiques servait d'ascenseur social au XIX ${ }^{e}$ et dans la première moitié du XX ${ }^{e}$ siècle. Au début du XXI siècle la francophonie associe les Bulgares aux institutions de l'Union européenne, à l'état de droit, aux acquis communautaires. Elle est susceptible de les rendre plus compréhensibles et accessibles aux Bulgares. Aussi, avait-elle contribué à l'instauration du climat social spécifique qui a rendu possible le sauvetage des juifs bulgares pendant la Seconde Guerre mondiale. Quelle en est la clé ?

31 En guise de conclusion, je voudrais citer de nouveau Jacques Émile Paris, ministre de France en Bulgarie au moment de la liquidation des écoles, en 1948 :

Le départ des religieux et religieuses français a donné lieu à des manifestations de sympathie émouvantes de la part des anciens élèves de nos écoles. La campagne d'excitation menée contre les établissements français à l'occasion de la fermeture des écoles étrangères est restée sans résultat. La parfaite dignité dont ont fait preuve nos religieux dans des circonstances aussi douloureuses semble avoir impressionné même ceux qui étaient les 
plus prévenus contre eux. Le prestige des congrégations enseignantes françaises reste intact en Bulgarie. C'est en très grande partie grâce à l'abnégation et au tact des Supérieurs des collèges et de tout le personnel enseignant que cette liquidation a pu s'effectuer dans ces conditions relativement satisfaisantes ${ }^{19}$.

32 J'ai eu la chance de côtoyer quelques élèves de la dernière promotion du collège Saint Augustin à Plovdiv: mon père et certains de ses condisciples ${ }^{20}$, et de saisir au vol l'esprit de cette ancienne jeunesse francophone noble. Son avenir étant peu prometteur vers la fin des années 1940 dans le tourbillon des changements politiques en Bulgarie, elle s'est dispersée pour semer la tolérance à la volée.

\section{BIBLIOGRAPHIE}

\section{Sources primaires}

Centre des archives diplomatiques de Nantes (CADN) - 585PO/1/28 Roustchouk Consulat, Honneurs liturgiques et protectorat (18 mai 1925-19 août 1927). Lettre de la légation de la République Française en Bulgarie (signée par Georges Picot), Sofia, à M. Mathieu, Consul de France à Roustchouk, du $1^{\text {er }}$ mars 1922.

Centre des archives diplomatiques de Nantes - Sofia Ambassade 644PO/1 (137) École de Sophia. Rapport sur l'expulsion du personnel français, 13.XI.1915.

Centre des archives diplomatiques de Nantes - Varna Consulat 720PO/1 (43).

Archives du ministère des Affaires étrangères (MAE) - CPC, vol. 6 (1890-1892) / P744 (p. 70, 71, 72 - 6 p.). Lettre du 7 avril 1891, adressée à M. le ministre des Affaires étrangères du Consulat de France à Philippopoli.

Archives du ministère des Affaires étrangères - microfilms P/1845. Lettre du Ministre de France à Sophia, Allizé, à S. Exc. M. Bourgeois, ministre des Affaires étrangères, 1905.

Archives du ministère des Affaires étrangères - Direction des consulats et des affaires commerciales (DCAC), Sous-Direction des Affaires commerciales (SDAC). Question des écoles en Bulgarie, Note pour la Direction des Affaires politiques, Paris, le 6 février 1906.

Archives du ministère des Affaires étrangères - microfilms P/18453. Lettre du Père Paul Journet, Directeur de l'École française des Pères Augustins de l'Assomption, à Varna, à M. Allizé, Ministre de France à Sophia, de 13 juin 1906.

Archives du ministère des Affaires étrangères - Archives diplomatiques, Z EUROPE, BULGARIE 1944-1949, CEuvres françaises en Bulgarie, Série Z Carton 76, 7 janvier 1948 - 28 juin 1949. Lettre de M. Jacques Émile Paris, ministre de France en Bulgarie à Son Excellence Monsieur le Ministre des affaires étrangères, le 19 novembre 1948 Liquidation des écoles françaises. 


\section{Sources secondaires}

ELDAROV, Svetlozar (2002). Katolitsite v Balgaria (1878-1989). Istorichesko izsledvane [Les catholiques en Bulgarie (1878-1989). Études historiques]. Sofia.

FLEURY, Alain (2001). Un collège français en Bulgarie (St Augustin, Plovdiv, 1884 - 1948). Paris :

L'Harmattan.

GALABERT, Victorin (2000). Journal. T. 2 (1867-1869). Sofia.

KOTCHEV, Nikolay (1988). « Roncali i prebivavaneto mu v Balgaria » [Angelo Roncali et son séjour en Bulgarie]. In Vtori mejdunaroden kongres po balgaristika [Deuxième congrès international d'études bulgares], Sofia, 23 mai - 3 juin 1986. Actes, t. 8 : Balgaria sled Osvobojdenieto (1878) [La Bulgarie après la Libération (1878)]. Sofia, 23-31.

« La Mission d'Orient de l'Assomption », (2010). Cahiers du Bicentenaire d'Alzon 2010, n 6. André Brombart a.a. (dir.). Série des Cahiers du Bicentenaire de la naissance du P. Emmanuel d'Alzon (1810-2010). En ligne : <http://www.assomption.org/fr/mediatheque /cahiers-du-icentenaire-1/ la-mission-d-orient-de-l-assomption> (31 mars 2015).

NIKOLOV, Nicola (1995). Chiprovskoto uchilishte [L'école de Chiprovtsi]. Sofia : Borina.

PÉRIER-MUZET, Jean-Paul, (2000). Notices biographiques des religieux de l'Assomption, 1850-2000. T. 2 , 1189 \& 1190. Rome : Maison généralice des Augustins de l'Assomption.

POMAREDE, Géraud, (1998). « Négocier près de la Sublime Porte ». In Lucien Bély, L'invention de la diplomatie. Moyen Age - Temps modèrnes. Paris : PUF. En ligne : <http://geraud.poumarede.free.fr/

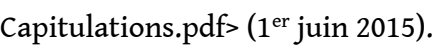

ROCHKÉVA, Renetta \& TZVETKOVA, Nadejda (2008). L'École française Notre-Dame de Sion à Roussé (1897-1948). Album bilingue. Roussé.

The National Statistical Institute of Bulgaria (2011 Census data). En ligne : <http:// www.eurel.info/spip.php?rubrique788\&lang=en> (31 mars 2015).

Société des Nations, Recueil des Traités, 1924. N701 Traité de Paix, signé à Lausanne le 24 juillet 1923. En ligne : <http://www.eurel.info/

IMG/pdf/gr_traite_lausanne.pdf> (31 mars 2015).

THOBIE, Jacques (2010). « L'importance des écoles dans la diffusion du français dans l'Empire ottoman au début du XX $\mathrm{X}^{\mathrm{e}}$ siècle ", Documents pour l'histoire du français langue étrangère ou seconde 38/39 | 2007. En ligne : <http://dhfles.revues.org/140> (31 mars 2015).

TODEV, Ilia (2002). « Avant-propos », Les Assomptionnistes et la Bulgarie 1862-2002, Exposition d'archives, Plovdiv, Alliance française, 11 novembre - 2 décembre 2002, Catalogue. Sofia.

ZAÏMOVA, Raïa, (2010). « Le français en Bulgarie dans le contexte de la politique culturelle de la France aux XIX ${ }^{\mathrm{e}}$ et XX $\mathrm{XX}^{\mathrm{e}}$ siècles ", Documents pour l'histoire du français langue étrangère ou seconde 38/39 | 2007. En ligne : <http://dhfles.revues.org/286> (31 mars 2015).

\section{NOTES}

1. En 1635 on construit un bâtiment spécial pour l'école de Chiprovtsi. Les élèves sont répartis en quatre classes. Ils apprennent à lire et à écrire en utilisant deux alphabets, le calcul, le catéchisme, la prière chrétienne et des choses utiles aux enfants de la ville. Pendant l'été de la 
même année, Petar Bogdan adresse une demande à Rome de lui envoyer une personne susceptible d'enseigner aux moines la logique, la philosophie et la théologie (Nikolov 1995 : 11).

2. Les capitulations de 1740 constituent, encore au milieu du XIXe siècle, la base du protectorat de la France en Orient (Poumarède 1998 : 85). Le Traité de Paix, signé à Lausanne le 24 juillet 1923, dans son article 28 déclare "l'abolition complète des Capitulations en Turquie à tous les points de vue » (Traité de Paix $1923: 8$ ). Le traité de commerce et de navigation entre la France et la Principauté de Bulgarie, signé à Sofia le 1er février 1906, remplace les Capitulations. Selon la Note d'A. Henry, «aucune des stipulations du nouveau traité n'avait pour conséquence d'apporter une modification à la situation actuelle des écoles françaises existant en Bulgarie et au régime dont elles bénéficient ». Cf. MAE - Direction des consulats et des affaires commerciales (DCAC), Sous-direction des Affaires commerciales (SDAC) - Question des écoles en Bulgarie, Note pour la direction des Affaires politiques, Paris, le 6 février 1906. "Rien n'a été changé en ce qui concerne le protectorat des catholiques et c'est, par suite, à la France qu'il incombe la charge d'assurer au regard de l'autorité Bulgare la liberté du culte catholique ». Lettre de la légation de la République Française en Bulgarie (signée par Georges Picot), Sofia, à M. Mathieu, consul de France à Roustchouk, du 1er mars 1922, CADN - 585PO/1/28 Roustchouk Consulat, Honneurs liturgiques et protectorat (18 mai 1925-19 août 1927). En bref, le traité de commerce et de navigation de 1906 qui remplace les capitulations de 1740 maintient le statu quo et la position privilégiée des écoles catholiques françaises en Bulgarie.

3. Victorin Galabert (1830-1885) est le fondateur de la Mission d'Orient. Il fait des études de médecine à la Faculté de Montpellier et obtient en 1854 un doctorat avec une thèse intitulée «Essai historique sur la variole». De 1858 à 1862, le P. Galabert est professeur au collège de Nîmes notamment en sciences naturelles.

4. MAE - microfilms P/1845, Lettre du ministre de France à Sophia, Allizé, à S. Exc. M. Bourgeois, ministre des Affaires étrangères, 1905.

5. La Triplice qui est la Triple-Alliance de l'Allemagne, l'Autriche-Hongrie et de l'Italie fondée le 20 mai 1882, cessa officiellement en mai 1915 lorsque l'Italie entra en guerre aux côtés des Alliés. La Bulgarie s'engage aux côtés des puissances centrales en octobre 1915.

6. CADN - Sofia Ambassade 644PO/1 (137) École de Sophia - Rapport sur l'expulsion du personnel français, 13.XI.1915.

7. Liste des écoles françaises en Bulgarie 1864-1948:1. École primaire gratuite Saint André à Philippopoli (Plovdiv), créée en novembre 1863 (début en janvier 1864) par le P. Galabert.

2. Collège Saint Augustin, créé en 1884 à Philippopoli (Plovdiv).

3. École des Sœurs de Saint Joseph de l'Apparition (1894-1948) à Philippopoli (Plovdiv).

4. École primaire de jeunes filles, à Yamboli, créée en 1888 par les Oblates de l'Assomption.

5. École primaire de garçons (petit séminaire-collège par la suite), à Yamboli, créée en 1889 par les Augustins de l'Assomption.

6. Collège de jeunes filles Saint André qui ouvre ses portes à Varna, en 1882.

7. École des Oblates de l'Assomption à Varna, installée depuis 1897.

8. École Française de garçons Saint Michel à Varna, fondée et dirigée par les Pères Augustins de l'Assomption. La première tentative de fondation date de l'année 1897. Enfin, l'école est autorisée en 1899 à titre personnel, au nom du Père Alexandre Chilier et comme école primaire seulement.

9. École mixte fondée en 1871 à Roustchouk (Roussé) par Monseigneur Hippolyte Agosto, évêque de Nicopolis.

10. École des Oblates de l'Assomption (1864-1896) pour filles à Roustchouk (Roussé).

11. École des Frères des Écoles chrétiennes (1890-1912) à Roustchouk (Roussé).

12. École française de jeunes filles Notre-Dame de Sion (1897-1948) l'internat, et l'externat Sancta Maria à Roustchouk (Roussé).

13. École française pour garçons Saint Joseph à Roussé (1918).

14. École des Sœurs de Saint Joseph de l'Apparition, 1891, Bourgas. 
15. École mixte (garçons et filles) des Sœurs Oblates de l'Assomption (1889-1891) à Bourgas.

16. Collège de garçons SS. Cyrille et Méthode à Sofia (Lozénetz), fondé en 1881 et dirigé par les Frères des Écoles chrétiennes.

17. Collège de jeunes filles Saint Joseph, fondé en 1880 et dirigé par les Sœurs de Saint Joseph de l'Apparition à Sofia.

8. MAE - microfilms P/18453. Lettre du Père Paul Journet, directeur de l'école française des Pères Augustins de l'Assomption, à Varna, à M. Allizé, ministre de France à Sophia, de 13 juin 1906.

9. CADN - Sofia Ambassade 644PO/1 (137). Version française de la convention scolaire entre la France et la Bulgarie, signée à Sophia le 17 décembre 1936 et publiée dans le Journal officiel $n^{\circ}$ 298 du 30 décembre 1936.

10. CADN - Varna Consulat $720 \mathrm{PO} / 1$ (43).

11. Cf. MAE - CPC, vol. 6 (1890-1892) / P744 (p. 70, 71, 72 - au total 6 p.) - Lettre du 7 avril 1891, adressée à $\mathrm{M}$. le ministre des Affaires étrangères du Consulat de France à Philippopoli. La lettre se rapporte aux écoles primaires de Bourgas et de Yamboli.

12. Son programme comprend les matières de commerce et comptabilité, les mathématiques appliquées au commerce et à la banque, les langues vivantes (français, bulgare, allemand), l'étude des marchandises, la géographie économique, l'histoire du commerce, le droit, l'économie politique, la religion et la morale pratique, enfin la dactylographie.

13. En terminale ou classe de philosophie, à côté de l'apologétique - 3e partie on apprend la philosophie (psychologie, logique, morale, métaphysique), l'histoire contemporaine, la géographie de la Bulgarie et de la France, les mathématiques (traité complet d'algèbre 3e partie calcul des dérivées, étude des maxima et minima, les fonctions algébriques et transcendantes), la géométrie descriptive, la méthode de Monge, la géométrie cotée, la cosmographie (éléments); Traité complet de physique : 3e partie - électricité statique et dynamique ; Traité complet de chimie: 3e partie - chimie organique ; Manipulations par les élèves ; Histoire naturelle : anatomie et physiologie animales; Hygiène; Manipulations par les élèves; Dessin géométrique et d'ornement.

14. Au cours de l'année scolaire 1941/1942 le temps scolaire des élèves de l'école est organisé comme suit: 134 heures d'enseignement du français, 254 heures d'enseignement des matières diverses enseignées en français, total : 388 heures. Le volume annuel d'heures d'enseignement devra respecter un total de 598 heures.

15. Horaire annuel d'enseignement du français : 93 heures ; matières diverses enseignées en français : 172 heures; enseignement dans d'autres langues que le français : 117 heures; volume annuel d'heures d'enseignement : 382 heures.

16. Cf. MAE-CADN, 720PO/1 - Plan d'études de l'école française de garçons, dirigée par les Pères Augustins de l'Assomption.

17. MAE, Archives diplomatiques, Z EUROPE, BULGARIE 1944-1949, Æuuvres françaises en Bulgarie, Série Z Carton 76, 7 janvier 1948 - 28 juin 1949.

18. En Bulgarie le français n'est ni langue maternelle, ni langue d'usage, ni langue officielle ou administrative, c'est une langue choisie et dans le cas bulgare exprime une appartenance volontaire à la langue française ou aux cultures francophones.

19. MAE - Archives diplomatiques, Z EUROPE, BULGARIE 1944-1949, Eeuvres françaises en Bulgarie, Série Z Carton 76, 7 janvier 1948 - 28 juin 1949, M. Jacques Emile Paris, Ministre de France en Bulgarie à Son Excellence Monsieur le Ministre des affaires étrangères, le 19 novembre 1948 a. s. Liquidation des écoles françaises.

20. Mon père, Angel Dimchev, juriste à Stara Zagora ; Kamen Hadjiyski, médecin à Plovdiv ; Atanas Tanev, un de mes professeurs de français au lycée Romain Rolland à Stara Zagora ; Michel Aghassian, anthropologue, professeur à l'EHESS-Paris, mais aussi Petar Uvaliev, connu aussi sous le pseudonyme de Pierre Rouve, diplomate, écrivain, journaliste, cinéaste, intellectuel bulgare, ayant terminé ses études au collège, en 1932 etc. 


\section{RÉSUMÉS}

Les conditions politiques et institutionnelles de la création des établissements catholiques français et de leur fonctionnement inégal mais efficace, en Bulgarie, entre 1864 et 1948, font l'objet de cette étude. À partir d'une liste exhaustive de ces écoles, établie pour la première fois, nous essayons de comprendre les raisons de leur implantation durable dans la vie sociale et culturelle des Bulgares malgré les considérations et les actes d'ordre politique, moral et religieux très souvent défavorables à leur égard. Ces écoles « catholiques » mais aussi « françaises » par la langue d'enseignement et parce qu'elles sont placées sous l'autorité française et subventionnées par la France (Secours aux établissements scolaires religieux et hospitaliers d'Orient, Département des affaires politiques - MAE, Service des œuvres françaises à l'étranger, l'Alliance française, l'Alliance israélite), semblent être à l'origine de la « francophonie d'appel » de la Bulgarie, aujourd'hui membre de l'OIF et dotée d'un réseau d'écoles secondaires bilingues (bulgare-français) - pépinières d'élites de sensibilité sociale et linguistique spécifiques.

This analysis focuses on the political and institutional conditions for the creation of French catholic schools and their unequal, but effective functioning in Bulgaria between 1864 and 1948 . The challenge is to try to understand the reasons for their lasting presence in the social and cultural life of the Bulgarians; this, despite political, moral and religious considerations and actions which are often unfavorable towards them. Being 'Catholic' but also 'French' according to the language of instruction and because they are placed under the French protectorate and are subsidized by France (Secours aux établissements scolaires religieux et hospitaliers d'Orient, Département des affaires politiques - MAE, Service des œuvres françaises à l'étranger, l'Alliance française, l'Alliance israélite) these schools seem to be the origin of the " francophonie d'appel » / 'Suggestive Francophonie' of Bulgaria, now a member of the IOF and possessing a network of bilingual High schools (Bulgarian-French) - a sort of nursery for elites with a specific social and linguistic sensitivity.

\section{INDEX}

Mots-clés : Bulgarie, écoles catholiques, collèges français, missions catholiques, congrégations enseignantes françaises, tolérance, dignité, valeurs morales françaises

Keywords : Bulgaria, Catholic schools, French High schools, Catholic missions, French teaching congregations, tolerance, dignity, French moral values

\section{AUTEUR}

\section{JULIETA VELICHKOVA-BORIN}

Université de Sofia Saint Clément d'Ohrid 